\title{
The shaping advantage of M-wire compared with conventional nickel-titanium rotary instruments in heavy curvature canal
}

Mika Tanaka-Sato

Kanagawa Shika Daigaku

Noriko Mutoh

Kanagawa Shika Daigaku

Kaori Shimojima

Kanagawa Shika Daigaku

Nobuyuki Tani-Ishii ( $\nabla$ n.ishii@kdu.ac.jp)

Kanagawa Shika Daigaku https://orcid.org/0000-0001-6326-284X

Research article

Keywords: M-wire, ProTaper NEXT, ProTaper Universal, shaping ability, apical curvature

Posted Date: August 10th, 2020

DOI: https://doi.org/10.21203/rs.3.rs-47617/v1

License: (c) (i) This work is licensed under a Creative Commons Attribution 4.0 International License.

Read Full License 


\section{Abstract}

Background The purpose of this study was to evaluate the shaping advantage of M-wire NiTi ProTaper NEXT (PTN) compared with a conventional NiTi ProTaper Universal (PTU) file in heavy curvature canal. The shaping ability was measured by the amount of canal cutting and transportation between the PTN and conventional PTU.

Methods Root canal shaping by the PTN and conventional PTU was classified into two experimental groups according to the final tip size, ISO \#25 or ISO \#40. Eighty-four J-shaped root canals $\left(10^{\circ}, 20^{\circ}, 30^{\circ}\right.$ apical curvature) in resin block were used.

Results After adjusting for the level and canal wall side, the mean transportation did not significantly decrease between the PTN and PTU with ISO \#25. Significantly less deviation occurred with the PTN and PTU between $10^{\circ}$ and $30^{\circ}$ using ISO \#40.

Conclusions The M-Wire NiTi PTN improves file flexibility and enables accurate canal shaping for heavy curvature canals.

\section{Background}

Rotary dental files with a NiTi alloy containing 56\% (wt) Ni and 44\% (wt) Ti have significantly changed root canal preparation. The NiTi alloy has a cubic crystal structure with an austenite phase above the shape recovery temperature (1). The austenite phase changes to martensite under force and temperature, but when the stress is released, the phase returns to austenite $(2,3)$. Consequently, the NiTi alloy has a shape memory effect and superelasticity without plastic deformation, which is advantageous for instruments used for root canal preparations.

Given the development of NiTi endodontic instruments targeted at root canals with complex anatomical configurations, the shape memory and superelastic properties of such NiTi instruments has helped prevent endodontic accidents (such as ledges, root canal perforations, apical transportation, and file fractures) $(4,5)$. Currently, unique heat and mechanical treatments can be used to complement the mechanical properties of NiTi alloys to improve flexibility and fatigue resistance.

In 2007, Sportwire LLC (Langley, OK, USA) developed a unique heat treatment process with the aim of producing a more flexible NiTi alloy with better fatigue resistance than conventional NiTi alloys (6). Additionally, Berendt developed a NiTi composition in which trace elements of less than $1 \%$ were added to produce a NiTi alloy (7). Under a heat treatment process, the M-Wire alloy converts to a rhombohedral (R) phase (intermediate temperature phase between austenite and martensite) and the elasticity changes with the change from austenite to martensite. Therefore, the flexibility of a NiTi endodontic file can be enhanced by modification $(8,9)$. Additionally, NiTi alloy converted to the $\mathrm{R}$ phase is conventionally produced by cutting; however, NiTi files are produced by a non-cutting rolling step, thereby increasing the file fatigue resistance and fracture resistance. 
The ProTaper NEXT (PTN) file used in this study is a next generation model of ProTaper Universal (PTU), which is a modification of the conventional NiTi alloy PTU and uses M-Wire. PTN was developed as a file suitable for severely curved root canals given its increased flexibility and fracture resistance. Compared with conventional NiTi PTU, the M-Wire NiTi PTN suppresses the occurrence of micro-cracks during root canal formation $(10,11)$. Root canal wall displacement after cutting (12-15), fatigue resistance (16-18), and file breakage resistance (19) of M-Wire PTN have been previously studied to determine the shaping ability. However, no report has objectively compared the different curvature canal range and shaping ability of M-Wire PTN with a conventional NiTi PTU in the same shaping system. The purpose of this study was to analyze the advantages of the M-Wire NiTi PTN and the relationship between the amount of the canal wall removed and canal center transportation compared with a conventional NiTi PTU.

\section{Methods}

\section{Simulated root canal block and experimental design}

Eighty-four simulated $\mathrm{J}$-shaped canal blocks with a curvature of $10^{\circ}, 20^{\circ}$ and $30^{\circ}$ (root apex size \#15, root canal length 19 mm, Nissin Plastic training Block S4-U1, Nissin, Kyoto) were used (Fig. 1).

We use a NiTi PTN file (Dentsply Sirona, Ballaigues, Switzerland) made by M-Wire and a conventional NiTi PTU file (Dentsply Sirona) as a control.

For root canal preparation, there were two final apical sizes for four groups, ISO \#25 (1 PTN, 2 PTU) and ISO \#40 (3 PTN, 4 PTU). Using a J-shaped canal block, measurements were made by classifying the blocks into 2 groups. The apical size and file taper (diameter increase amount / $\mathrm{mm}$ ) of each NiTi file were PTN X1 (17/04 (tip diameter/taper), X2 (25/06), X3 (30/07), X4 (40/06), and PTU F1 (20/04), F2 (25/06), F3 (30/06), and F4 (40/06), which were used to set the final apical size to ISO \#25 and \#40.

Three curvature blocks $\left(10^{\circ}, 20^{\circ}, 30^{\circ}\right.$; each $\left.n=7\right)$ were used, giving 21 total canal blocks for each of the ISO \#25 (1:PTN, 2:PTU) and ISO \#40 (3:PTN, 4:PTU) groups. Therefore, 84 canal blocks were tested.

\section{Root canal preparation}

Before starting root canal preparation with the M-wire PTN and conventional PTU files, flare formation (straight line) in the upper third of all root canal orifice openings and smooth file guide path creation (glide path) up to the root apex were performed. Flare formation was performed using PTN SX and PT SX files (Dentsply Sirona), which are used for straight line formation only. Furthermore, the glide path was formed up to \#15 using a ISO\#15K stainless-steel file. All NiTi files were washed with purified water using a dedicated engine (X Smart plus, Dentsply Sirona), and $1 \mathrm{~mL}$ of EDTA-gel Glide (Dentsply Sirona) was applied to the NiTi files.

Root canal preparation using PTN and PTU files was performed by a single dentist with more than 7 years of experience using NiTi rotary files. The following procedure was performed and files were exchanged after every five root canal formations. 
1. ISO \#25 PTN: Using two $X 1$ and $X 2$ files sequentially, select the $X$ Smart plus PTN mode ( $300 \mathrm{rpm}$, $2.0 \mathrm{Ncm}$ ), perform root canal cleaning when exchanging files, and reach the working length of the $\mathrm{X} 2$ file to complete the root canal formation.

2. ISO \#25 PTU: Using S1, S2, F1 and F2 files in sequence, select the PT mode (250 rpm, 3.0-1.0 Ncm), perform root canal cleaning when exchanging files, and let the $\mathrm{F} 2$ file reach its working length.

3. ISO \#40 PTN: Using X1, X2, X3, and X4 files in sequence, select the PTN mode (300 rpm, $2.0 \mathrm{Ncm}$ ), perform root canal cleaning when exchanging files, and let the $\mathrm{X} 4$ file reach its working length.

4. ISO \#40 PTU : Using S1, S2, F1, F2, F3, and F4 files in sequence, select the PT mode (250 rpm, 3.0$1.0 \mathrm{Ncm}$ ), perform root canal cleaning when exchanging files, and save the F4 file after reaching its working length.

\section{Evaluation of the canal shaping}

The amount of resin removed in the outer and inner side of the curved root canal was compared. A stereoscopic microscope Olympus SZX16 and a digital camera DP71 (Olympus, Tokyo) were used for the measurement. The transparent root canal models before and after root canal preparation was superimposed with digital images, and the resulting image was transferred to a PC and measured. The measurement points were 1, 2, 3, 4, and $5 \mathrm{~mm}$ from the apex, and the increase in the root canal width on the outer and inner side was measured and statistical processing was performed (Fig. 2). Root canal transportation was measured as the amount of root canal wall cutting in the outer and inner sides of the curved root canal, and the median displacement of the root canal before and after preparation was measured.

\section{Statistical analysis}

The amount of root canal wall cutting and root canal transportation was measured using statistical analysis using one-way analysis of variance and a multiple comparison test using Bonferroni-Dunn test. The level of significance was test at $p<0.05$.

\section{Results}

The root canal shaping ability was evaluated according to the amount of resin removed and the root canal transportation. In experimental groups 1 to 4 , the amount of resin removed was shown by comparing M-wire PTN and conventional PTU for each curvature $\left(10^{\circ}, 20^{\circ}, 30^{\circ}\right.$; Figs. $\left.3,4,5\right)$. The root canal transportation was classified by PTN and PTU file (Tables 1 and 2).

\section{Shaping ability of the final apical size ISO \#25}

The removal of resin by PTN decreased in both the inner and outer side in the ISO \#25 curvatures of $10^{\circ}$ to $30^{\circ}$ compared with the PTU $\otimes F i g .3 a, 4 a, 5 a \rrbracket$. Alternatively, the amount of resin removed by PTU on the inner and outer sides was small at $10^{\circ}$ to $20^{\circ}$ curvatures. The amount of resin removed by PTU a little 
increased at the $30^{\circ}$ curvature: the apex side was $1 \mathrm{~mm}$ on the outer side, and the apical side is $4 \mathrm{~mm}$ and $5 \mathrm{~mm}$ on the inner side (Fig. $5 \mathrm{~b}$ ).

The change in the root canal transportation for both PTN and PTU was within $0.05 \mathrm{~mm}$ for all root canals with a curvature of $10^{\circ}$ to $30^{\circ}$ (Table 1 ).

\section{Shaping ability of the final apical size ISO \#40}

Even with ISO \#40 and PTN, the amount of resin removed from the inner and outer sides was less than those in the PT group for all root canals with a curvature of $10^{\circ}$ to $30^{\circ} \otimes \mathrm{Fig} .3 \mathrm{~b}, 4 \mathrm{~b}, 5 \mathrm{~b}$. For ISO \#40 and PTN, the amount of resin removed was significantly less than the PTU group at $1-5 \mathrm{~mm}$ on the inner side with a curvature of $10^{\circ}$ (Fig.3b) and $1 \mathrm{~mm}$ on the outer side at $30^{\circ}$ (Fig.5b). The amount of resin removed by PTN only increased from 4 to $5 \mathrm{~mm}$ on the inner side compare with outer side at $30^{\circ}$ (Fig. $5 \mathrm{~b}$ ). This tendency was similar to that of the group. The amount of resin removed by PTU with ISO \#40 significantly increased by $1 \mathrm{~mm}$ on the apex side for all a curvatures, and the cutting amount on the inner side increased from 4 to $5 \mathrm{~mm}$ on the apex side冈p<0.05区(Fig.5b).

The change in the root canal transportation was less than $0.05 \mathrm{~mm}$ in the $10^{\circ}$ to $20^{\circ}$ curvatures using PTN, but $0.06 \mathrm{~mm}$ at the apex 4 to $5 \mathrm{~mm}$ into the root with a $30^{\circ}$ curvature. The transportation further significantly increased to $0.08 \mathrm{~mm} \rrbracket \mathrm{p}<0.05 \rrbracket$ (Table2).

Alternatively, the root canal transportation of PTU showed a displacement of $0.1 \mathrm{~mm}$ or more on the outer side of the apex side at $1 \mathrm{~mm}$ in all the root canals with a curvature of $10^{\circ}$ to $30^{\circ}$. In the PTU, the canal transportation on the inner side increased to $0.07 \mathrm{~mm}$ and $0.08 \mathrm{~mm}$ at 3 and $4 \mathrm{~mm}$, respectively, even with a curvature of $10^{\circ}$, and the inner side displacement of the root canal of $30^{\circ}$ also increased to 0.07 $\mathrm{mm}$ and $0.08 \mathrm{~mm}$ at 4 and $5 \mathrm{~mm}$, respectively (Table 2 ).

\section{Discussion}

The mechanical properties of the NiTi file depends on the processing method of the file and the surface finishing method, but it has been reported that the change in crystal structure by the heat treatment process affects the flexibility of NiTi alloys (20-23). The PTU used in this study has super-elasticity by the stress-induced transformation of the martensitic phase with a monoclinic crystal structure to the austenite phase, which is the cubic crystal structure of a conventional NiTi alloy (24). Alternatively, PTN, which is a NiTi alloy processed into M-wire by heating and cooling, coexists with austenite, martensite, and $\mathrm{R}$ phases, and has a monoclinic martensite phase and $\mathrm{R}$ phase (16-18). Because the elastic modulus is less than that of the austenite phase, the initial flexibility is improved. Additionally, the M-wire NiTi alloy is more flexible than the conventional NiTi alloy because the austenite phase undergoes a martensitic transformation due to the stress on the file when forming a curved root canal. The stress load on the file is reduced, which reduces file corruption (19). 
In this study, the usefulness of the M-wire NiTi alloy was analyzed by comparing the cutting characteristics of PTN and PTU under a stress load (formation of a curved root canal). The root canal formation using root canal models with different apex curvatures of $10^{\circ}$ to $30^{\circ}$ were analyzed with final apical sizes of ISO \#25 and ISO \#40. There was a large difference in the displacement of the root canal wall between the groups. In the ISO \#25 group, there was no significant difference in the amount of resin removed or canal transportation associated with the change in the bending angle in PTN and PTU.

In the ISO \#25 group, the amount of resin removed by PTN and PTU was 1 to $5 \mathrm{~mm}$ from the apex of the inner and outer bays for curved root canal angles of $10^{\circ}, 20^{\circ}$, and $30^{\circ}$. The measured value was less than $0.05 \mathrm{~mm}$. Furthermore, the root canal transportation was less than $0.05 \mathrm{~mm}$ and both files could form the root canal and maintain the original anatomical root canal morphology. The NiTi file with a ISO \#25 tip showed that the shaping ability of the conventional and M-wire type NiTi alloy files were similar and useful for root canal preparation of curved root canals between $10^{\circ}$ to $30^{\circ}$.

Alternatively, in the ISO \#40 group, the amount of resin removed significantly changed depending on the root canal curvature in the PTN and PTU file. The PTU increased the lateral displacement by $1 \mathrm{~mm}$ from the apex, and $4 \mathrm{~mm}$ and $5 \mathrm{~mm}$ from the apex in the inside of the root canal between $10^{\circ}$ to $30^{\circ}$. The risk of transportation and canal perforation in the curved root canal was observed. However, the PTN maintains the original root canal morphology when the amount of resin removed in the inner and outer sides was 1 to $3 \mathrm{~mm}$ and from the apex is $0.1 \mathrm{~mm}$ or less between $10^{\circ}$ to $30^{\circ}$ canals. Alternatively, for the $30^{\circ}$ curvature canal, the inner side cutting of $4 \mathrm{~mm}$ and $5 \mathrm{~mm}$ from the apex tended to be as high as that of the PTU. The PTN obtained by converting the NiTi alloy into the R phase by heat processing improved the root canal followability and suppressed the transport on the outer bay side of the apex. It is necessary to be careful of accidents, such as strip perforation during root canal formation on the inner side, when using the $30^{\circ}$ curvature.

Because the PTN and PTU used in this study are similar file systems for complete root canal formation with multiple lines, the flexibility of the M-Wire NiTi alloy is useful for proper root canal formation. Additionally, the M-wire type NiTi alloy single file preparation system (Reciproc, WaveOne file) completes root canal formation with only one file, the root canal wall displacement is less than the conventional NiTi PTU, and the root canal transportation is reduced. Additionally, the M-wire PTN is reported to retain anatomical morphology $(23,24)$. Finally, the M-wire PTN has excellent fatigue resistance and torsion resistance regardless of the difference in file systems used for root canal preparation.

\section{Conclusion}

The amount of canal wall removed and transported by M-wire ISO \#40 PTN was less than that of conventional ISO \#40 PTU in $10^{\circ}$ to $30^{\circ}$ curvature canals and the original root canal form was maintained, although no significant difference in the shaping ability for curvature canals by both PTN and PTU of ISO \#25. The M-Wire PTN improves file flexibility and enables accurate canal shaping for wide and heavy curved canals 


\section{Abbreviations}

NiTi: nickel-titanium ; PTN: ProTaper Next; PTU: ProTaper Universal

\section{Declarations}

\section{Acknowledgements}

The authors deny any conflicts of interest related to this study. We thank Ashleigh Cooper, PhD, from Edanz Group (https://en-author-services.edanzgroup.com/) for editing a draft of this manuscript.

\section{Author's Contributors:}

NT, MT, NM, and KS were responsible for the concept, design, and implementation of the work, analyzed and interpretation of data. MT and NM were major contributors in writing the manuscript. NT was funding acquisition, writing-review and editing the manuscript. All authors read and approved the final manuscript.

\section{Funding:}

This study was supported by a part of Grant-in-Aid for Scientific Research from the Ministry of Education, Culture, Sports, Science and Technology of Japan (B) (No. 20390437). NT was funding acquisition

\section{Availability of data and materials}

The datasets used and/or analyzed during the current study are available from the corresponding author on reasonable request.

\section{Ethics approval and consent to participate}

Not applicable

\section{Consent for publications}

Not applicable. 


\section{Competing interest:}

The authors deny any conflict of interest related to this study.

\section{References}

1. Thompson SA. An overview of nickel-titanium alloys used in dentistry. Int Endod. 2000;33:297-310.

2. Lee JH, Park JB, Andreason GF, Lakes RS. Thermomechanical study of NiTi alloys. J of Biomed Materials Res. 1988; 22: 573-88.

3. Serene TP, Adams JD, Saxena A. Nickel-Titanium instruments: Applications Endodontics. St Lous MO, USA: Ishiyaku Euro America Inc.

4. Walia HM, Brantley WA, Gerstein H. An initial investigation of the bending and torsional properties of Nitinol root canal files. J Endod 1988; 14: 346-51.

5. Kazemi RB, Stenman E, Spångberg LS. A comparison of stainless steel and nickel-titanium H-type instruments of identical design: torsional and bending tests.

Oral Surg Oral Med Oral Pathol Oral Radiol Endod 2000; 90: 500-6.

6. Zinelis S, Darabara M, Takase T, Ogane K, Papadimitriou GD. The effect of thermal Treatment on the resistance of nickel-titanium rotary files in cyclic fatigue. Oral Surg Oral Med Oral Pathol Oral Radiol Endod. 2007 ; 103:843-7.

7. Berendet C. Method of preparing Nitinol for use in manufacturing instrument with improved fatigue resistance. US patent Application 20070072147 Al.

8. Alapati SB, Brantley WA, lijima M, Clark WAT, Phil D, KovarikL, et al. Metallurgical characterization of a newnickel-itanium wire for rotary endodontic instruments. J Endod 2009;35:1589-93.

9. Pereira ESJ, Peixoto IFC, Viana ACD, Oliveira II, Gonzalez BM,Buono VTL, et al. Physical and mechanical properties of athermomechanically treated NiTi wire used in themanufacture of rotary endodontic instruments. Int Endod J 2012; 45:469-74.

10. De-Deus G, Belladonna FG, Souza EM, Silva EJ, Neves Ade A, Alves H, Lopes RT, Versiani MA.De-Deus $\mathrm{G}$, et al. Micro-computed Tomographic Assessment on the Effect of ProTaper Next and Twisted File Adaptive Systems on Dentinal Cracks. J Endod. 2015;41:1116-9.

11. Saha SG, Vijaywargiya N, Saxena D, Saha MK, Bharadwaj A, Dubey S.Saha SG, et al. Evaluation of the incidence of microcracks caused by Mtwo and ProTaper Next rotary file systems versus the self-adjusting file: A scanning electron microscopic study.

J Conserv Dent. 2017;20:355-359.

12. Hiran-us S, Pimkhaokham S, Sawasdichai J, Ebihara A, Suda H. Shaping ability of ProTaper NEXT, ProTaper Universal and iRace files in simulated S-shaped canals. Aust Endod J. 2016; 42: 32-6.

13. Gagliardi J, Versiani MA, de Sousa-Neto MD, Plazas-Garzon A, Basrani B. Evaluation of the Shaping Characteristics of ProTaper Gold, ProTaper NEXT, and ProTaper Universal in Curved Canals. J Endod. 2015; 41:1718-24.

14. Wu H, Peng C, Bai Y, Hu X, Wang L, Li C. Shaping ability of ProTaper Universal, WaveOne and ProTaper Next in simulated L-shaped and S-shaped root canals. BMC Oral Health. 2015;15:27. doi: 10.1186/s12903-015-0012. 
15. Tavanafar S, Gilani PV, Saleh AM, Schäfer E. Shaping Ability of ProTaper Universal, ProTaper NEXT and WaveOne Primary in Severely Curved Resin Blocks. J Contemp Dent Pract. 2019; 20: 363-369.

16. Özyürek T, Yılmaz K, Uslu G.Özyürek T, et al. The effects of autoclave sterilization on the cyclic fatigue resistance of ProTaper Universal, ProTaper Next, and ProTaper Gold nickel-titanium instruments. Restor Dent Endod. 2017; 42: 301-308.

17. Topçuoğlu HS, Topçuoğlu G, Akti A, Düzgün S.Topçuoğlu HS, et al. In Vitro Comparison of Cyclic Fatigue Resistance of ProTaper Next, HyFlex CM, OneShape, and ProTaper Universal Instruments in a Canal with a Double Curvature. J Endod. 2016; 42: 969-71.

18. Uygun AD, Kol E, Topcu MK, Seckin F, Ersoy I, Tanriver M.Uygun AD, et al. Variations in cyclic fatigue resistance among ProTaper Gold, ProTaper Next and ProTaper Universal instruments at different levels. Int Endod J. 201; 49: 494-9.

19. Fernández-Pazos G, Martín-Biedma B, Varela-Patiño P, Ruíz-Piñón M, Castelo-Baz P.Fernández-Pazos G, et al. Fracture and deformation of ProTaper Next instruments after clinical use.J Clin Exp Dent. 2018; 10:e1091-e1095.

20. E.S. Pereira, R.O. Gomes, A.M. Leroy, et al. Mechanical behavior of M-wire and conventional NiTi wire used to manufacture rotary endodontic instruments

Dent Mater. 2013; 29: e318-e324

21. J.L. Gutmann, Y. GaoAlteration in the inherent metallic and surface properties of nickel-titanium root canal instruments to enhance performance, durability and safety: a focused review. Int Endod J. 2012; 45: 113-128

22. L.C. Braga, A.C. Silva, V.T. Buono, M.G. Bahia. Impact of heat treatments on the fatigue resistance of different rotary nickel-titanium instruments

J Endod. 2014; 40; 1494-1497

23. E.S.J. Pereira, I.F.C. Peixoto, A.C.D. Viana, et al.Physical and mechanical properties of a thermomechanically treated $\mathrm{NiTi}$ wire used in the manufacture of rotary endodontic instruments. Int Endod J. 2012; 45: 469-474

24. A.S. Câmara, R.C. Martins, A.C.D. Viana, et al.Flexibility and torsional strength of ProTaper and ProTaper Universal rotary instruments assessed by mechanical tests

J Endod. 2009; 35: 113-116

\section{Tables}

Due to technical limitations, full-text HTML conversion of the tables could not be completed. However, the tables can be downloaded and accessed as a PDF in the supplementary files

\section{Figures}


a)

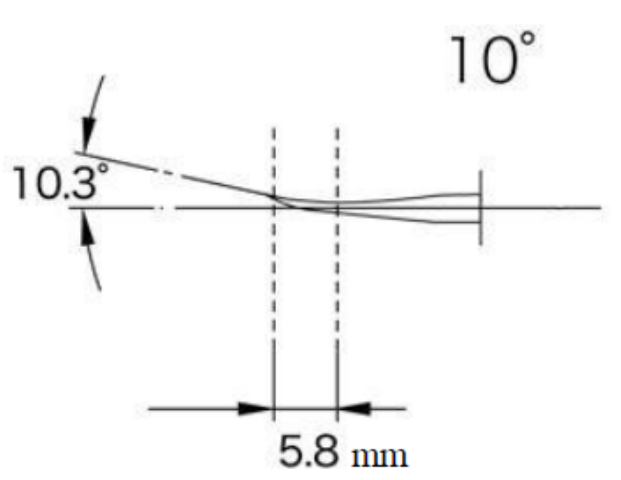

b)

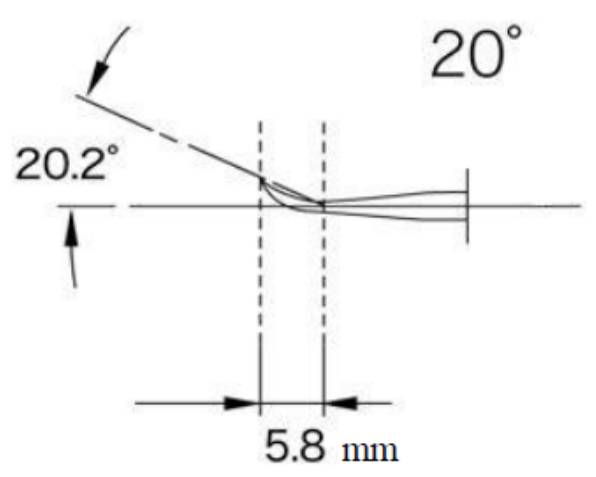

c)

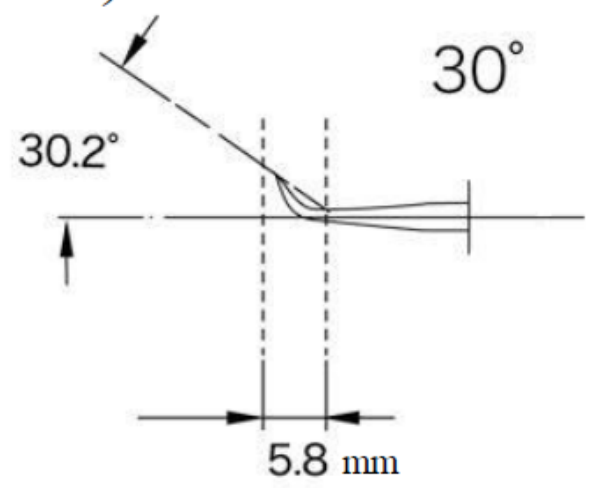

Figure 1

J-shaped simulated root canals made of clear resin with apical size of $0.15 \mathrm{~mm}$ (ISO \#15) and (a) $10^{\circ}$, (b) $20^{\circ}$, and (c) $30^{\circ}$ apical curvatures.

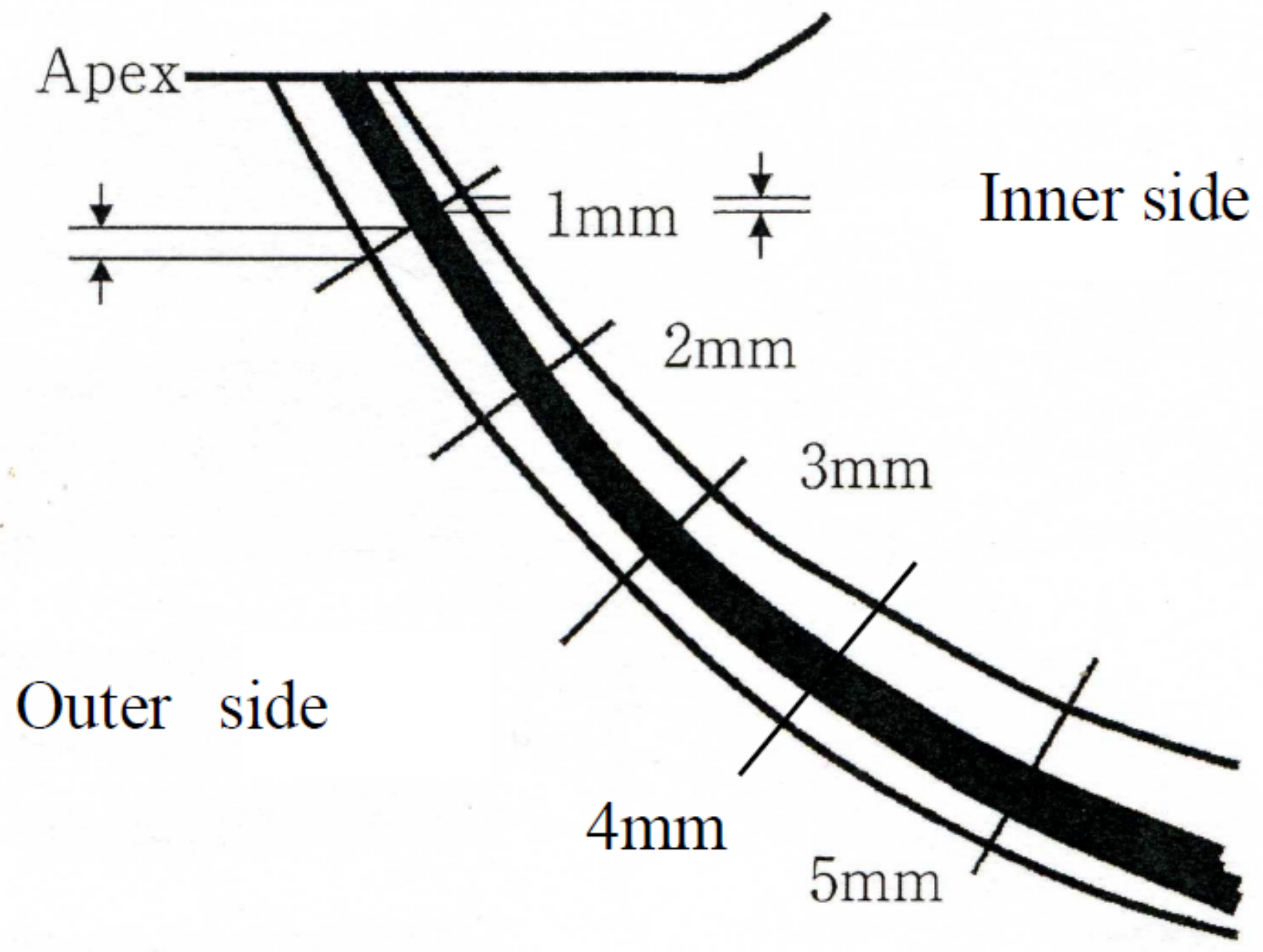

Figure 2 
Pre- and post-instrumentation images were superimposed and the difference between the canal configuration before and after instrumentation were measured in each of the five traced levels.

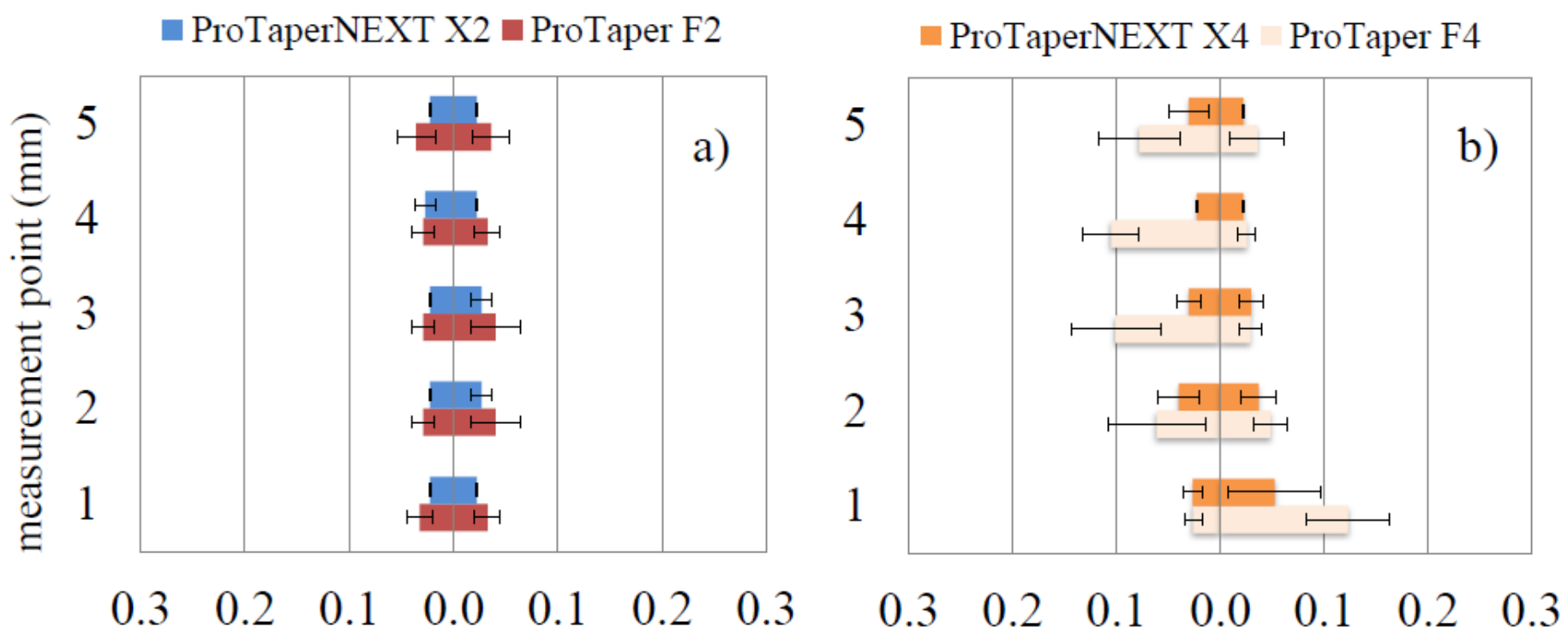

Figure 3

Total amount of removed resin $(\mathrm{mm})$ at the different levels after root canal preparation for $10^{\circ}$ apical curvature canals by (a) ISO \#25 PTN, ISO \#25 PTU, and (b) ISO \#40 PTN, ISO \#40 PTU.

- ProTaperNEXT X2 - ProTaper F2

ProTaperNEXT X4 ProTaper F4

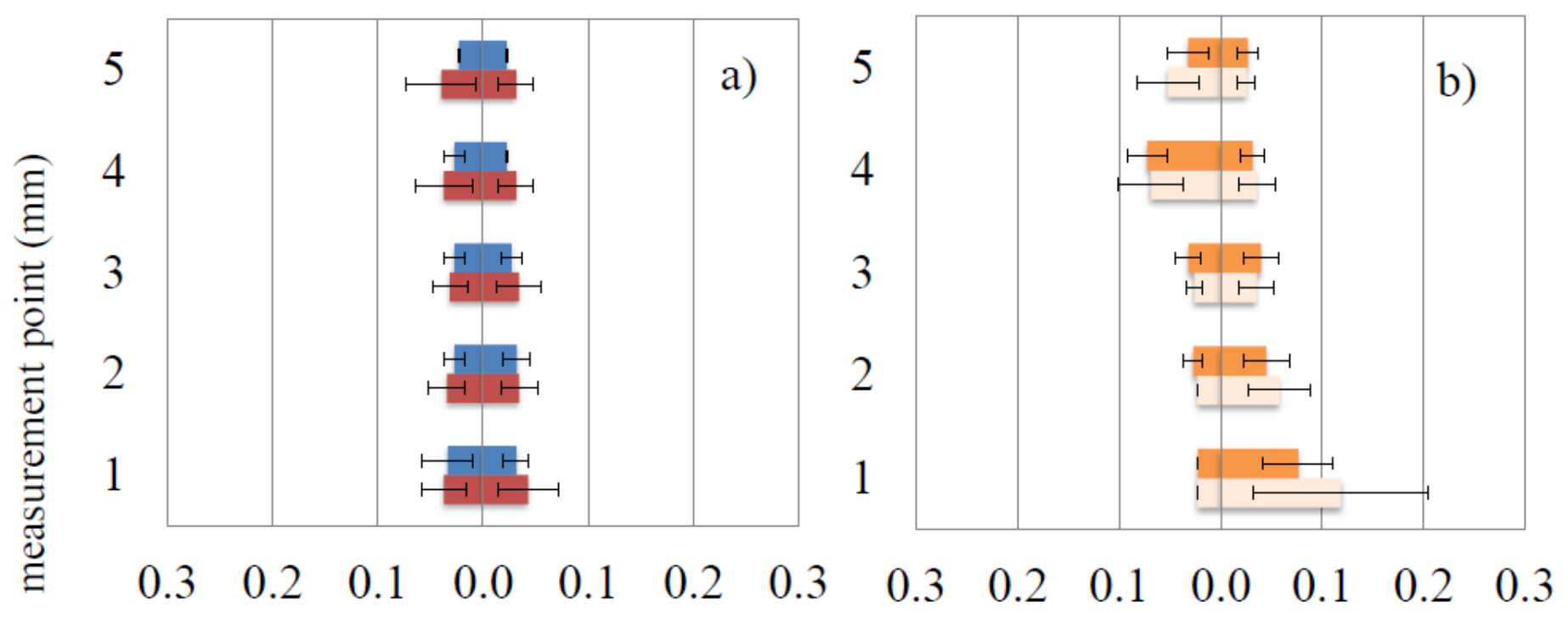

Figure 4

Total amount of removed resin $(\mathrm{mm})$ at the different levels after root canal preparation for $20^{\circ}$ apical curvature canals by (a) ISO \#25 PTN, ISO \#25 PTU, and (b) ISO \#40 PTN, ISO \#40 PTU. 


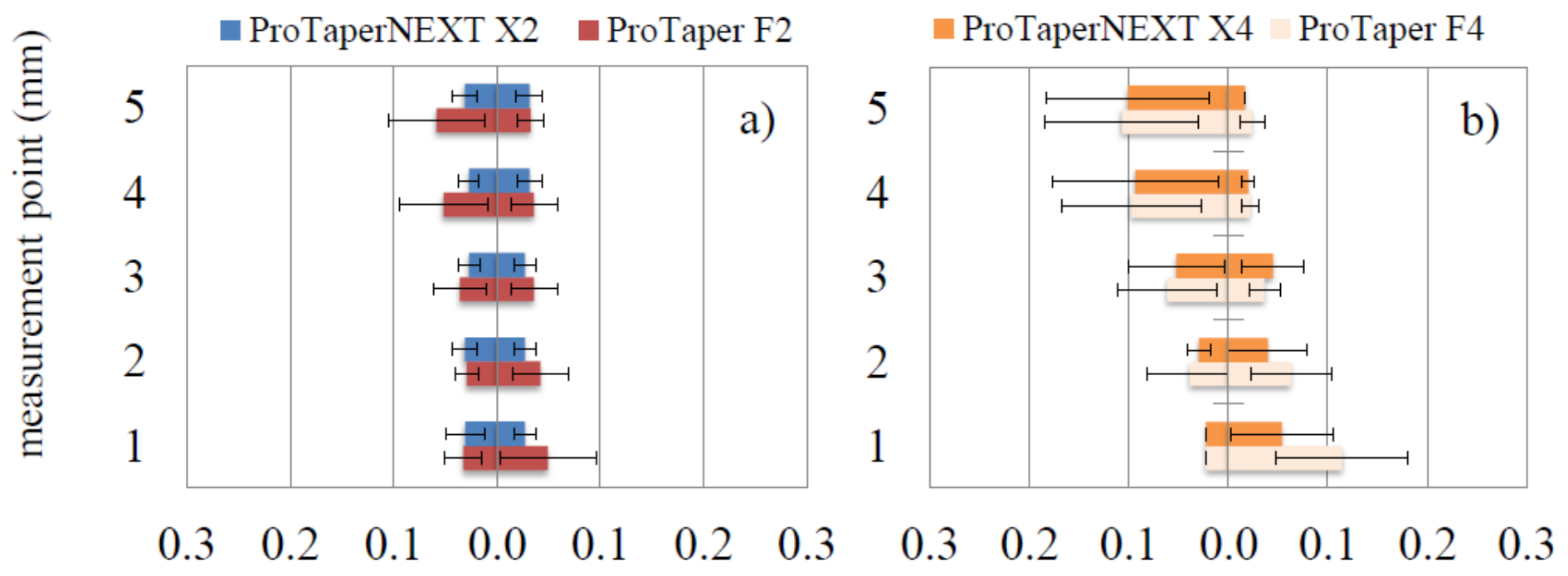

Figure 5

Total amount of removed resin $(\mathrm{mm})$ at the different levels after root canal preparation for $30^{\circ}$ apical curvature canals by (a) ISO \#25 PTN, ISO \#25 PTU, and (b) ISO \#40 PTN, ISO \#40 PTU.

\section{Supplementary Files}

This is a list of supplementary files associated with this preprint. Click to download.

- renamedbbd5c.pdf

- renamed717f4.pdf 